\title{
Early Versus Late Laparoscopic Cholecystectomy in Patients with Acute Gallstone Pancreatitis
}

\author{
Ashish Sharma $^{1}$ (I) $\cdot$ Apoorva Madapu ${ }^{2} \cdot$ Jigisha Rakholiya ${ }^{3} \cdot$ Shivy Sharma ${ }^{4} \cdot$ Anil Jha $^{5}$
}

Accepted: 13 January 2021 / Published online: 22 January 2021

(C) The Author(s) 2021

\begin{abstract}
The laparoscopic cholecystectomy is the definite treatment of acute gallstone pancreatitis, but the timing remains controversial. We aimed to evaluate trends, prevalence, and outcomes of laparoscopic cholecystectomy amongst patients with acute gallstone pancreatitis in index hospitalization done at different time intervals. We performed a retrospective study using Nationwide Inpatient Sample (years 2003-2014) with adult hospitalizations with acute gallstone pancreatitis who underwent laparoscopic cholecystectomy using ICD-9-CM codes and divided them into subgroups of those who underwent immediately ( $72 \mathrm{~h}$ ), early (1 week), and delayed (> 1 week) laparoscopic cholecystectomy. The weighted analysis using chi-square, paired Wilcoxon-rank-sum test, Cochran-Armitage trend test, and multivariable survey logistic regression analysis was performed to evaluate prevalence and outcomes. There was a total of 582,406 acute gallstone pancreatitis admissions of which 291,701 (50.09\%) patients had laparoscopic cholecystectomy $(p<0.0001)$. Prevalence of immediate laparoscopic cholecystectomy 114,629 (40.55\%) showed incremental trend (32.61 to 49.82\%) compared to early 149,931 (53.03\%) (57.83 to 46.59\%), and late laparoscopic cholecystectomy 18,158 (6.42\%) (9.56 to $3.59 \%$ ) from 2003 to 2014. Immediate and early laparoscopic cholecystectomy had lower prevalence and odds of mortality [0.24\% versus $0.37 \%$ versus $2.46 \%$; aOR-immediate: 0.614 ; aOR-early: $0.615 ; p<0.0001$ ] , morbidity [1.36\% versus $3.64 \%$ versus $27.57 \%$; aOR-immediate: 0.088 ; aOR-early: $0.161 ; p<0.0001]$, disability [62.47\% versus $70.15 \%$ versus 84.26\%; aOR-immediate: 0.584 ; aOR-early: $0.68 ; p<0.0001$ ], discharge to non-home [7.07\% versus $13.04 \%$ versus 31.84\%; \%; aOR-immediate: 0.346 ; aOR-early: $0.473 ; p<0.0001$ ], hospital cost $\$ 39,466$ versus $\$ 52,018$ versus $\$ 129,317(p<0.0001)$ and stay 3 days versus 6 days versus 19 days $(p<0.0001)$ than delayed laparoscopic cholecystectomy. The immediate laparoscopic cholecystectomy showed better outcomes; more prospective studies with large numbers of patients should be planned to evaluate the beneficial effects of immediate cholecystectomy in severe acute pancreatitis hospitalizations.
\end{abstract}

Keywords Acute pancreatitis management $\cdot$ Gallstone pancreatitis $\cdot$ Biliary pancreatitis $\cdot$ Laparoscopic cholecystectomy trend and prevalence $\cdot$ Gallstone pancreatitis guideline $\cdot$ Acute pancreatitis management guideline

This article is part of the Topical Collection on Medicine

Ashish Sharma

Ashishsharma1412@gmail.com

Apoorva Madapu

Apporva.madapu@gmail.com

Jigisha Rakholiya

Jigirakholiya@gmail.com

Shivy Sharma

Shivysharma130@gmail.com

Anil Jha

Dranil3jha@gmail.com
1 FACP, Department of Internal Medicine, Yuma Regional Medical Center, 2400 S Ave A, Yuma, AZ, USA

2 Huntsville Hospital, Huntsville, AL, USA

3 Mayo Clinic Rochester, Rochester, MN, USA

4 Department of Internal Medicine, Marshfield Clinic Health System, Marshfield, WI, USA

5 Department of Internal Medicine, Lawrence General Hospital, 1 General St, Lawrence, MA 01841, USA 


\section{Introduction}

The annual incidence rate for acute pancreatitis is 4.9 to 35 per 100,000 cases. Acute gallstone pancreatitis is the most common etiology for acute pancreatitis and accounts for 40-70\% of the total case, but only 3 to $7 \%$ of patients with gallstones develop acute pancreatitis [1,2]. Acute pancreatitis incidence is increasing with an increasing rate of obesity and gallstone [3]. Small gallstone less than $5 \mathrm{~mm}$ has shown to be associated with a higher risk of acute gallstone pancreatitis [4]. Treatment for acute gallstone pancreatitis includes initially conservative and supportive treatment and then definite treatment of underlying cause in the form of either laparoscopic cholecystectomy or endoscopic retrograde cholangiopancreatography (ERCP) with sphincterotomy if cholecystectomy cannot be performed for any reason. Laparoscopic cholecystectomy is a wellknown and proven definitive treatment for acute gallstone pancreatitis in the absence of which there is $25-30 \%$ risk of recurrent acute pancreatitis, cholecystitis, cholangitis, and other gallstone-related complication such as biliary colic within 6 to 18 weeks $[5,6]$. Multiple studies including randomized control trial and systematic review with meta-analysis have observed that early cholecystectomy (EC) in patients is feasible, safe, and effective with low risk of complications in carefully selected patients with mild acute biliary pancreatitis based on different clinical severity prediction risk tools such as Ranson criteria, Acute Physiology and Chronic Health Evaluation (APACHE II), Bedside severity index in pancreatitis (BISAP), and Modified CT Severity Index (MCTSI). Most studies also observed decreased hospital length of stay, decrease rate of readmissions, and recurrent gallstone-related complications in patients with mild acute gallstone pancreatitis who had early and index admission cholecystectomy [7-10]. American College of Gastroenterology Guideline recommends cholecystectomy before discharge to prevent recurrence in mild biliary pancreatitis [11]. UK Guidelines for the Management of Acute Pancreatitis 2005 recommends doing cholecystectomy in the same admission [12].

Similarly, the International Association of Pancreatology (IAP) and the American Pancreatic Association (APA) evidence-based guidelines for the management of acute pancreatitis recommend cholecystectomy during index admission for mild biliary pancreatitis and further states high risk of readmission for recurrent biliary events, especially recurrent biliary pancreatitis with interval cholecystectomy (GRADE 1C, strong agreement) [7]. Most gastroenterology society guidelines around the world acknowledge the benefit of endorsed EC. However, the ideal timeline of intervention has not been established in guidelines because of various definitions of early cholecystectomy in clinical studies. The guidelines differ on the timeline for cholecystectomy and range from as early as 24 to $48 \mathrm{~h}$ of admission to a few weeks. Most guidelines agree and strongly recommend early cholecystectomy in index admission; lack of clear consensus on cholecystectomy's timing is also one of the reasons for poor adherence to guidelines. In this paper, we aim to study the trends, prevalence, and outcomes of different timeline interventions classified as immediate, early, and delayed laparoscopic cholecystectomy amongst patients with acute gallstone pancreatitis.

\section{Methods}

We gathered the national inpatient sample data from January 2003 to December 2014 from the Agency for Healthcare Research and Quality's Healthcare Cost and Utilization Project (HCUP). It is the largest publicly available all-payer inpatient care database in the USA and contains dischargelevel data provided by states that participate in the HCUP. This administrative dataset contains data on approximately 8 million hospitalizations in 1000 hospitals that are chosen to approximate a $20 \%$ stratified sample of all the US community hospitals, representing more than $95 \%$ of the national population. Detailed information on NIS is available at http://www. hcup-us.ahrq.gov/db/nation/nis/nisdde.jsp.

\section{Study Population}

We used the 9th revision of the International Classification of Diseases, clinical modification (ICD-9-CM) code to identify adult patients admitted to hospital with a primary diagnosis of acute gallstone pancreatitis (ICD-9-CM code 577.8). Similarly, patients who underwent laparoscopic cholecystectomy were identified using ICD-9 procedure code 51.23 and divided them into subgroups of who underwent immediate (within $72 \mathrm{~h}$ ), early (within a week), and delayed (>1 week) laparoscopic cholecystectomy. We used ICD-9-CM codes to identify independent predictors (covariates), including the comorbidities of hypertension, diabetes mellitus, obesity, hypercholesterolemia, acquired immunodeficiency syndrome, ischemic heart disease, end-stage renal disease, chronic kidney disease, drug abuse, alcohol abuse/dependence and withdrawal, smoker (current/past), and chronic pancreatitis. Similarly, we identified complications like hypercalcemia, acute renal failure, shock, systemic inflammatory response syndrome, ascites, pleural effusion, respiratory distress/failure, and portal vein thrombosis. We also recognized the procedures like ERCP, abdominal ultrasound, CT scan of the abdomen, cholecystostomy, blood transfusions, and total parenteral nutrition done. Age $<18$ years and admissions with missing data for age, gender, and race were excluded. The sample size was based on the available data. 


\section{Patient and Hospital Characteristics}

Patient characteristics of interest were gender, age, race, insurance status, and concomitant diagnoses, as mentioned above. The race was defined by White (referent), African-American, Hispanic, Asian or Pacific Islander, and Native American. Insurance status was defined by Medicare (referent), Medicaid, private insurance, and other/self-pay/no charge. We defined the severity of comorbid conditions using Deyo's modification of the Charlson Comorbidity Index (Supplementary Table 1). Facilities were considered to be teaching hospitals if they have an American Medical Association-approved residency program, are a member of the Council of Teaching Hospitals, or have full-time equivalent interns and residents to a patient's ratio $\geq 0.25$. The HCUP NIS contains data on total charges for each hospital in the databases, which represents the amount that hospitals billed for services.

\section{Outcomes}

The primary aim of this study was to evaluate the characteristics of acute gallstone pancreatitis patients who underwent immediate, early, and delayed laparoscopic cholecystectomy and the prevalence of its complications. The secondary aim was to evaluate the outcomes like morbidity, mortality, disability (loss of function), discharge disposition, length of stay (LoS), and cost of hospitalization associated with immediate laparoscopic cholecystectomy (years 2003-2014). The comparison of disability/loss of function was investigated by All Patient Refined Diagnosis Related Groups (APR-DRGs) severity $[28,29]$. APR-DRGs were assigned using software developed by $3 \mathrm{M}$ Health Information Systems, where score 1 indicates minor loss of function, 2-moderate, 3-major, 4 extreme loss of function. Morbidity defined as the length of stay $>11$ days (90th percentile) and discharge other than home (short-term hospital, skilled nursing facility, or intermediate care facility). Discharge disposition was defined by discharge to home versus non-home.

\section{Statistical Analysis}

All statistical analyses were performed using the weighted survey methods in SAS (version 9.4). The $p$ values $<0.05$ were considered significant. Univariate analysis of differences between categorical variables was tested using the chi-square test, and analysis of differences between continuous variables (age, LoS, and cost of hospitalization) was tested using paired Student's $t$ test. Mixed-effects survey logistic regression models with weighted analysis were used to evaluate the predictors of immediate laparoscopic cholecystectomy in the acute gallstone pancreatitis hospitalization during 20032014. We included demographics (age, gender, race), patient-level hospitalization variables (admission day, primary payer, admission type, Median Household Income Category), hospital-level variables (hospital region, teaching versus nonteaching hospital, hospital bed size), comorbidities and concurrent conditions, complications, and Charlson's Comorbidity Index (CCI). The goodness of fit of the model was evaluated by the $c$-value.

\section{Results}

\section{Disease Hospitalizations}

We found a total of 582,406 acute pancreatitis hospitalizations due to gallstone pancreatitis from 2003 to 2014 after excluding patients with missing data for age, race, sex, and outcomes. Out of 582,406 hospitalizations, 291,701 (50.09\%) patients had laparoscopic cholecystectomy during the same hospitalization. Out of the same hospitalization cholecystectomy, the prevalence of immediate (within $72 \mathrm{~h}$ ), early (within one week), and late ( $>1$ week) laparoscopic cholecystectomy was 114,629 (40.55\%), 149,931 (53.03\%), and 18,158 (6.42\%), respectively.

\section{Prevalence Trend}

We analyzed the trend of immediate, early, and late laparoscopic cholecystectomy in acute gallstone pancreatitis. As shown in Fig. 1, the trend of immediate laparoscopic cholecystectomy has increased, and early and late laparoscopic cholecystectomy has decreased from 2003 to 2014 (immediate laparoscopic cholecystectomy, $32.61 \%$ in 2003 to $49.82 \%$ in 2014; early laparoscopic cholecystectomy, $57.83 \%$ in 2003 to $46.59 \%$ in 2014; and late laparoscopic cholecystectomy, $9.56 \%$ in 2003 to $3.59 \%$ in 2014; pTrend $<0.0001$ ).

\section{Demographics, Patient and Hospital Characteristics, and Comorbidities}

Patients with the age group of 18-55 years have higher prevalence of immediate laparoscopic cholecystectomy utilization $(56.47 \% ; p<0.0001)$ as compared to early and late laparoscopic cholecystectomy $(46.30 \%$ versus $41.00 \% ; p<0.0001)$. Whereas patients with age group $>55$ years have higher prevalence of late laparoscopic cholecystectomy $(59.00 \%$; $p<$ $0.0001)$ as compared to immediate and early laparoscopic cholecystectomy $(43.53 \%$ versus $53.70 \%$; $p<0.0001)$. In females, the prevalence of immediate laparoscopic cholecystectomy $(67.06 \%)$ was higher in comparison to early $(61.59 \%)$ and late laparoscopic cholecystectomy $(54.62 \%)(p<0.0001)$. Whereas in males, prevalence of late laparoscopic cholecystectomy $(45.38 \%)$ was more than immediate $(32.94 \%)$ and early laparoscopic cholecystectomy $(38.41 \%)(p<0.0001)$. 
Immediate, early and late laparoscopic cholecystectomy amongst patients with acute gallstone cholecystitis in pancreatitis.

70

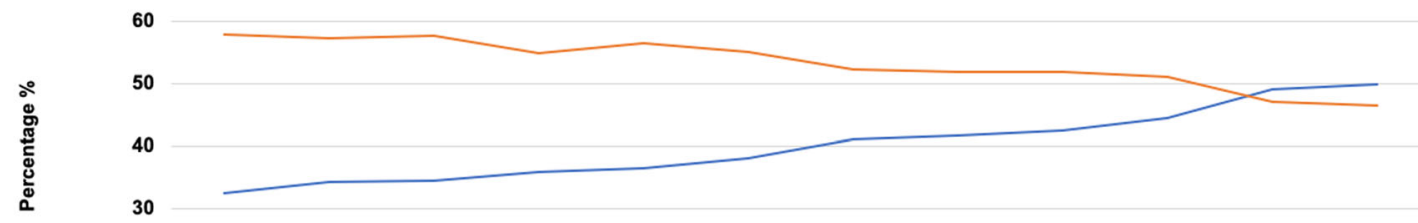

20

10

o

- Immediate laparoscopic cholecystectomy

- Early laparoscopic cholecystectomy

- Late laparoscopic cholecystectomy

\begin{tabular}{|c|c|c|c|c|c|c|c|c|c|c|c|}
\hline 2003 & 2004 & 2005 & 2006 & 2007 & 2008 & 2009 & 2010 & 2011 & 2012 & 2013 & 2014 \\
\hline $\mathbf{3 2 . 6 1}$ & 34.41 & 34.53 & 36.01 & $\mathbf{3 6 . 6 2}$ & $\mathbf{3 8 . 0 5}$ & $\mathbf{4 1 . 2 1}$ & $\mathbf{4 1 . 7}$ & $\mathbf{4 2 . 4 8}$ & $\mathbf{4 4 . 5}$ & $\mathbf{4 9 . 0 4}$ & $\mathbf{4 9 . 8 2}$ \\
\hline $\mathbf{5 7 . 8 3}$ & $\mathbf{5 7 . 2 1}$ & $\mathbf{5 7 . 7 7}$ & $\mathbf{5 4 . 9 3}$ & $\mathbf{5 6 . 4 5}$ & $\mathbf{5 5 . 1 3}$ & $\mathbf{5 2 . 3 5}$ & $\mathbf{5 1 . 9 2}$ & $\mathbf{5 1 . 8 5}$ & $\mathbf{5 1 . 0 9}$ & $\mathbf{4 7 . 0 2}$ & $\mathbf{4 6 . 5 9}$ \\
\hline $\mathbf{9 . 5 6}$ & $\mathbf{8 . 3 8}$ & $\mathbf{7 . 7 1}$ & $\mathbf{9 . 0 6}$ & $\mathbf{6 . 9 3}$ & $\mathbf{6 . 8 2}$ & $\mathbf{6 . 4 5}$ & $\mathbf{6 . 3 8}$ & $\mathbf{5 . 6 6}$ & $\mathbf{4 . 4 1}$ & $\mathbf{3 . 9 3}$ & $\mathbf{3 . 5 9}$ \\
\hline
\end{tabular}

Fig. 1 Immediate, early, and late laparoscopic cholecystectomy among patients with acute gallstone cholecystitis in pancreatitis

Immediate laparoscopic cholecystectomy was more commonly performed in Native American [(48.85\%) versus whites (40.71\%), African-American (34.35\%), Hispanic (42.67\%), and Asian/Pacific Islander $(41.49 \%)(p<0.0001)]$, in patients with private insurance $[(46.01 \%)$ versus Medicare $(33.70 \%)$, Medicaid (42.49\%), and other/self-pay/no charge $(41.22 \%)(p$ $<0.0001)$ ], having elective admissions [(54.51\%) versus emergency admissions $(39.74 \%)(p<0.0001)]$, weekday admissions [(41.76\%) versus weekends $(37.32 \%)(p<0.0001)]$, in rural hospitals $[(44.49 \%)$ versus urban non-teaching (41.19\%), and urban teaching hospital location $(38.84 \%)(p$ $<0.0001)$ ], and in west region [ $(49.98 \%)$ versus northeast (29.65\%), Midwest (42.88), and south region $(38.65 \%)(p<$ 0.0001)].

Patients with comorbidities like obesity (immediate, $18.68 \%$ versus early, $18.13 \%$; late, $16.44 \%$; $p<0.0001)$ and current or past smoker (immediate, $19.19 \%$ versus early, $17.82 \%$; late, $15.21 \%$; $p<0.0001)$ were having higher prevalence of immediate laparoscopic cholecystectomy as compared to early and late laparoscopic cholecystectomy. Patients with comorbidities like hypertension (immediate, $39.65 \%$ versus early, $49.17 \%$; late, $54.50 \%$; $p<0.0001$ ), diabetes (immediate, $16.53 \%$ versus early, $20.61 \%$, late, $26.43 \%$; $p<0.0001$ ), hypercholesterolemia (immediate, $6.21 \%$ versus early, 7.35\%; late, 7.54\%; $p<0.0001$ ), drug abuse (immediate, $1.12 \%$ versus early, $1.43 \%$; late, $2.14 \%$; $p<0.0001$ ), alcohol abuse (immediate, $2.89 \%$ versus early, $4.47 \%$; late, $8.46 \% ; p<0.0001)$, acquired immunodeficiency syndrome (immediate, 0.10\%; versus early, 0.13\%; late, $0.48 \%$; $p<$ 0.0001 ), ischemic heart disease (immediate, $9.20 \%$ versus early, $13.78 \%$; late, $16.74 \% ; p<0.0001)$, end-stage renal disease (immediate, $0.62 \%$ versus early, $1.05 \%$; late, $2.13 \%$; $p<$ 0.0001 ), chronic kidney disease (immediate, $2.83 \%$ versus early, 3.69\%; late, 5.89\%; $p<0.0001$ ), alcohol withdrawal (immediate, $0.09 \%$ versus early, 0.24\%; late, $0.85 \%$; $p<$ 0.0001 ), and chronic pancreatitis (immediate, $0.72 \%$ versus early, $1.20 \%$; late, $3.04 \%$; $p<0.0001)$ were having lower prevalence of immediate laparoscopic cholecystectomy as compared to early and late laparoscopic cholecystectomy. Prevalence of need of blood transfusion (immediate, 2.68\%; versus early, $4.46 \%$; late, $14.57 \%$; $p<0.0001)$ and total parenteral transfusion (immediate, $0.92 \%$; versus early, $2.39 \%$; late, $19.29 \% ; p<0.0001)$ were lower in patients undergoing immediate laparoscopic cholecystectomy as compared to early and late laparoscopic cholecystectomy.

Prevalence of complications like hypercalcemia (immediate, $0.18 \%$; versus early, $0.27 \%$; late, $0.53 \%$; $p<$ 0.0001 ), acute renal failure (immediate, $3.07 \%$; versus early, 5.78\%; late, 17.51\%; $p<0.0001$ ), shock (immediate, $0.27 \%$; versus early, $0.37 \%$; late, $3.59 \% ; p<0.0001$ ), systemic inflammatory response syndrome (immediate, $1.33 \%$; versus early, $2.34 \%$; late, $10.35 \%$; $p<0.0001$ ), ascites (immediate, 1.14\%; versus early, 1.94\%; late, $6.50 \% ; p<0.0001$ ), pleural effusion (immediate, $0.04 \%$, versus early, $0.08 \%$; late, $0.54 \% ; p<0.0001)$, respiratory distress and failure (immediate, $1.27 \%$; versus early, $2.09 \%$; late, $11.39 \%$; $p<0.0001)$, and portal vein thrombosis (immediate, $0.04 \%$, versus early, $0.07 \%$; late, $0.60 \%$; $p<0.0001)$ were lower in patients undergoing immediate laparoscopic cholecystectomy as compared to early and late laparoscopic cholecystectomy (Table 1).

\section{The Outcomes}

Table 2 has mentioned the outcomes of immediate, early, and late laparoscopic cholecystectomy in patients with acute gallstone pancreatitis. The patients who underwent immediate laparoscopic cholecystectomy had lower prevalence of morbidity (immediate, $1.36 \%$; versus early, 3.64\%; late, $27.57 \%$; $p<0.0001$ ), mortality (immediate, $0.24 \%$; versus early, $0.37 \%$; late, $2.46 \% ; p<0.0001)$, major/extreme disability (immediate, 62.47\%; versus early, 70.15\%; late, $84.26 \%$; $p$ 
Table 1 Outcomes and healthcare burden of immediate, early, and late laparoscopic cholecystectomy in patients with acute gallstone pancreatitis

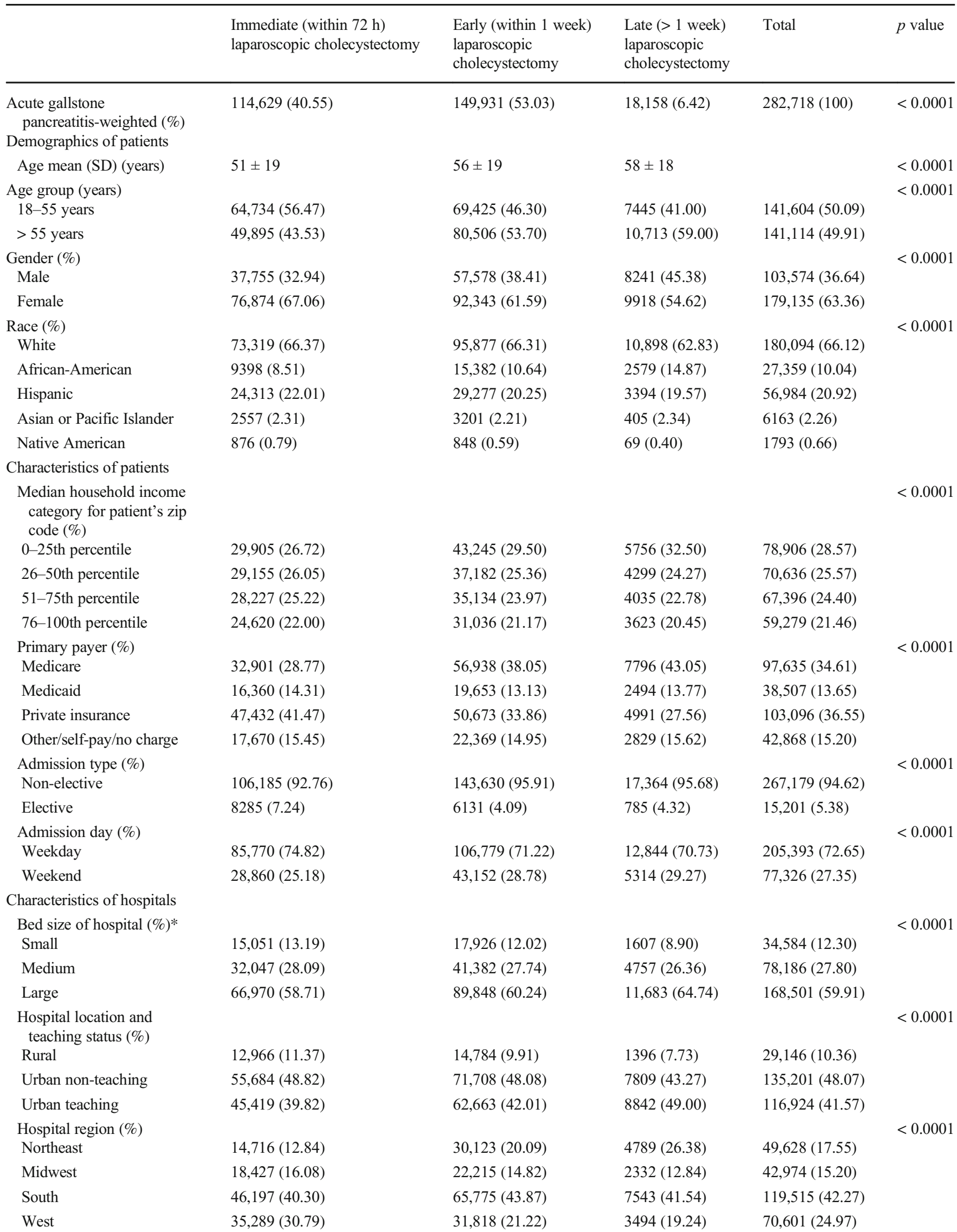


Table 1 (continued)

Immediate (within $72 \mathrm{~h}$ )

laparoscopic cholecystectomy
Early (within 1 week)

laparoscopic

cholecystectomy
Total

$p$ value

( $>1$ week)

laparoscopic

cholecystectomy
Comorbidities of patients (\%)

\begin{tabular}{|c|c|c|c|c|c|}
\hline Hypertension & $45,448(39.65)$ & $73,719(49.17)$ & $9897(54.50)$ & $129,064(45.65)$ & $<0.0001$ \\
\hline Diabetes & $18,946(16.53)$ & $30,897(20.61)$ & $4799(26.43)$ & $54,642(19.33)$ & $<0.0001$ \\
\hline Obesity & $21,410(18.68)$ & $27,190(18.13)$ & $2985(16.44)$ & $51,585(18.25)$ & $<0.0001$ \\
\hline Hypercholesterolemia & $7117(6.21)$ & $11,018(7.35)$ & $1368(7.54)$ & $19,503(6.90)$ & $<0.0001$ \\
\hline Drug abuse & $1284(1.12)$ & $2139(1.43)$ & $388(2.14)$ & $3811(1.35)$ & $<0.0001$ \\
\hline Alcohol abuse & $3307(2.89)$ & $6705(4.47)$ & $1537(8.46)$ & $11,549(4.09)$ & $<0.0001$ \\
\hline Current or past smoker & 21,997 (19.19) & $26,716(17.82)$ & $2762(15.21)$ & $51,475(18.21)$ & $<0.0001$ \\
\hline $\begin{array}{l}\text { Acquired immunodeficiency } \\
\text { syndrome (AIDS) }\end{array}$ & $116(0.10)$ & $202(0.13)$ & $86(0.48)$ & $404(0.14)$ & $<0.0001$ \\
\hline Ischemic heart disease & $10,540(9.20)$ & $20,657(13.78)$ & $3040(16.74)$ & $34,237(12.11)$ & $<0.0001$ \\
\hline End-stage renal disease & $707(0.62)$ & $1577(1.05)$ & $387(2.13)$ & $2671(0.95)$ & $<0.0001$ \\
\hline Chronic kidney disease & $3249(2.83)$ & $5538(3.69)$ & $1070(5.89)$ & $9857(3.49)$ & $<0.0001$ \\
\hline Alcohol withdrawal & $108(0.09)$ & $366(0.24)$ & $153(0.85)$ & $627(0.22)$ & $<0.0001$ \\
\hline Chronic pancreatitis & $826(0.72)$ & $1793(1.20)$ & $553(3.04)$ & $3172(1.12)$ & $<0.0001$ \\
\hline \multicolumn{6}{|l|}{ omplications (\%) } \\
\hline Hypercalcemia & $211(0.18)$ & $402(0.27)$ & $96(0.53)$ & $709(0.25)$ & $<0.0001$ \\
\hline Acute renal failure & $3525(3.07)$ & $8670(5.78)$ & $3179(17.51)$ & $15,374(5.44)$ & $<0.0001$ \\
\hline Shock & $304(0.27)$ & $554(0.37)$ & $653(3.59)$ & $1511(0.53)$ & $<0.0001$ \\
\hline $\begin{array}{l}\text { Systemic inflammatory } \\
\text { response syndrome }\end{array}$ & $1523(1.33)$ & $3501(2.34)$ & $1879(10.35)$ & $6903(2.44)$ & $<0.0001$ \\
\hline Ascites & $1308(1.14)$ & $2902(1.94)$ & $1179(6.50)$ & $5389(1.91)$ & $<0.0001$ \\
\hline Pleural effusion & $45(0.04)$ & $125(0.08)$ & $99(0.54)$ & $269(0.10)$ & $<0.0001$ \\
\hline $\begin{array}{l}\text { Respiratory distress } \\
\text { and respiratory failure }\end{array}$ & $1453(1.27)$ & $3140(2.09)$ & $2068(11.39)$ & $6661(2.36)$ & $<0.0001$ \\
\hline Portal vein thrombosis & $45(0.04)$ & $104(0.07)$ & $109(0.60)$ & $258(0.09)$ & $<0.0001$ \\
\hline \multicolumn{6}{|l|}{ rocedures (\%) } \\
\hline $\begin{array}{l}\text { Endoscopic retrograde } \\
\text { cholangiopancreatography }\end{array}$ & $3329(2.90)$ & $8952(5.97)$ & $1587(8.74)$ & $13,868(4.91)$ & $<0.0001$ \\
\hline Abdominal ultrasonography & 1935 (1.69) & $3536(2.36)$ & $681(3.75)$ & $6152(2.18)$ & $<0.0001$ \\
\hline $\begin{array}{l}\text { Computed tomography } \\
\text { scan of abdomen }\end{array}$ & $1534(1.34)$ & $3985(2.66)$ & $1023(5.64)$ & $6542(2.31)$ & $<0.0001$ \\
\hline Cholecystostomy & $117(0.10)$ & $156(0.10)$ & $218(1.20)$ & 491(0.17) & $<0.0001$ \\
\hline Blood transfusion & $3067(2.68)$ & $6693(4.46)$ & $2646(14.57)$ & $12,406(4.39)$ & $<0.0001$ \\
\hline Total parenteral nutrition & $1056(0.92)$ & $3587(2.39)$ & $3503(19.29)$ & $8146(2.88)$ & $<0.0001$ \\
\hline \multicolumn{6}{|l|}{ Comorbidity Index (CCI) } \\
\hline 0 & $78,598(68.57)$ & $90,632(60.45)$ & $8574(47.22)$ & $177,804(62.89)$ & \\
\hline 1 & $23,152(20.20)$ & $35,088(23.40)$ & $4833(26.61)$ & $63,073(22.31)$ & \\
\hline 2 & $7267(6.34)$ & $13,128(8.76)$ & $2473(13.62)$ & $22,868(8.09)$ & \\
\hline 3 & $3021(2.64)$ & $5866(3.91)$ & $1202(6.62)$ & $10,089(3.57)$ & \\
\hline 4 & $1336(1.17)$ & $2837(1.89)$ & $522(2.88)$ & $4695(1.66)$ & \\
\hline 5 & $1254(1.09)$ & $2380(1.59)$ & $554(3.05)$ & $4188(1.48)$ & \\
\hline
\end{tabular}

Percentage in brackets are column \% indicating direct comparison between immediate, early, and late laparoscopic cholecystectomy in patients with acute gallstone pancreatitis

*Bed size of hospital indicates number of hospital beds which varies depends on hospital location (rural/urban), teaching status (teaching/non-teaching), and region (Northeast/Midwest/Southern/Western)

$<0.0001$ ), discharge other than home (immediate, $7.07 \%$; versus early, $13.04 \%$; late, $31.84 \%$; $p<0.0001)$, length of stay (immediate, 4 days; versus early, 6 days; late, 19 days; $p<$ 0.0001 ), and cost of hospitalization (immediate, $\$ 39,466$; 
Table 2 Outcomes of immediate, early, and late laparoscopic cholecystectomy in patients with acute gallstone pancreatitis

\begin{tabular}{|c|c|c|c|c|c|}
\hline & $\begin{array}{l}\text { Immediate (within } 72 \text { h) } \\
\text { laparoscopic } \\
\text { cholecystectomy }\end{array}$ & $\begin{array}{l}\text { Early (within } 1 \text { week) } \\
\text { laparoscopic } \\
\text { cholecystectomy }\end{array}$ & $\begin{array}{l}\text { Late (>1 week) } \\
\text { laparoscopic } \\
\text { cholecystectomy }\end{array}$ & Total & $p$ value \\
\hline Acute gallstone pancreatitis-weighted (\%) & $114,629(40.55)$ & $149,931(53.03)$ & $18,158(6.42)$ & $282,718(100)$ & $<0.0001$ \\
\hline Morbidity* & $1555(1.36)$ & $5455(3.64)$ & $5006(27.57)$ & $12,016(4.25)$ & $<0.0001$ \\
\hline Mortality & $271(0.24)$ & $559(0.37)$ & $446(2.46)$ & $1276(0.45)$ & $<0.0001$ \\
\hline \multicolumn{5}{|l|}{ Disability } & \multirow[t]{3}{*}{$<0.0001$} \\
\hline Minor/moderate disability & $42,815(37.53)$ & $44,347(29.85)$ & $2769(15.74)$ & $89,931(32.09)$ & \\
\hline Major/extreme disability & $71,259(62.47)$ & $104,242(70.15)$ & $14,828(84.26)$ & $190,329(67.91)$ & \\
\hline \multicolumn{5}{|l|}{ Discharge disposition } & \multirow[t]{3}{*}{$<0.0001$} \\
\hline Discharge to home & $8072(7.07)$ & $19,443(13.04)$ & $5627(31.84)$ & $33,142(11.80)$ & \\
\hline Discharge other than home $\mathrm{e}^{\#}$ & $106,090(92.93)$ & $129,619(86.96)$ & $12,048(68.16)$ & 247,757 (88.20) & \\
\hline Length of stay \pm SE (days) & $4 \pm 0.02$ & $6 \pm 0.02$ & $19 \pm 0.26$ & & $<0.0001$ \\
\hline Cost of hospitalization $\pm \mathrm{SE}(\$)$ & $39,466 \pm 276$ & $52,018 \pm 271$ & $129,317 \pm 2678$ & & $<0.0001$ \\
\hline
\end{tabular}

*Morbidity: length of stay $\geq 11$ days (90th percentile) + discharge other than home

\# Discharge other than home: discharge to short-term hospital, skilled nursing facility, or intermediate care facility

versus early, $\$ 52,018$; late, $\$ 129,317 ; p<0.0001)$ than early and late laparoscopic cholecystectomy (Table 3 ).

\section{Regression Model Derivation}

We have noticed lower chances of morbidity (immediate, aOR: 0.09; 95\%CI: 0.07-0.10; $p<0.0001$; early, aOR: 0.16; 95\% CI: $0.14-0.18 ; p<0.0001)$, mortality (immediate, aOR: 0.61; 95\%CI: 0.38-0.99; $p<0.0001$; early, aOR: 0.61; 95\% CI: $0.41-0.93 ; p<0.0001$ ), disability (immediate, aOR: 0.58; 95\%CI: 0.52-0.65; $p<0.0001$; early, aOR: 0.68; 95\%CI: $0.61-0.76 ; p<0.0001)$, and discharge predisposition (immediate, aOR: 0.35; 95\%CI: $0.31-0.39 ; p<0.0001$; early,
aOR: $0.47 ; 95 \%$ CI: $0.42-0.53 ; p<0.0001)$ in immediate and early laparoscopic cholecystectomy as compared to late laparoscopic cholecystectomy. The $c$-statistic which is used to validate the accuracy of the regressions was $>0.5$ which indicates a good model (morbidity, 0.92; mortality, 0.94; disability, 0.75; and discharge predisposition, 0.87) (Table 2).

\section{Discussion}

In our study, we analyzed national inpatient trend and the prevalence of immediate (within $72 \mathrm{~h}$ ), early (within 1 week), and late (after 1 week) laparoscopic cholecystectomy in acute gallstone

Table 3 Outcomes associated with immediate, early, and late laparoscopic cholecystectomy in patients with acute gallstone pancreatitis

\begin{tabular}{|c|c|c|c|c|}
\hline & $\begin{array}{l}\text { Morbidity* } \\
\text { Odds ratio } \\
\text { (confidence } \\
\text { interval) ( } p \text { value) }\end{array}$ & $\begin{array}{l}\text { Mortality } \\
\text { Odds ratio } \\
\text { (confidence } \\
\text { interval) ( } p \text { value) }\end{array}$ & $\begin{array}{l}\text { Disability } \\
\text { Odds ratio } \\
\text { (confidence } \\
\text { interval) ( } p \text { value) }\end{array}$ & $\begin{array}{l}\text { Discharge disposition } \\
\text { Odds ratio (confidence } \\
\text { interval) ( } p \text { value) }\end{array}$ \\
\hline Late ( $>1$ week) laparoscopic cholecystectomy & Reference & & & \\
\hline $\begin{array}{l}\text { Immediate (within } 72 \mathrm{~h} \text { ) } \\
\text { laparoscopic cholecystectomy }\end{array}$ & $\begin{array}{l}0.09(0.07-0.10) \\
\quad(p<0.0001)\end{array}$ & $\begin{array}{l}0.61(0.38-0.99) \\
\quad(p<0.0001)\end{array}$ & $\begin{array}{l}0.58(0.52-0.65) \\
\quad(p<0.0001)\end{array}$ & $0.35(0.31-0.39)(p<0.0001)$ \\
\hline $\begin{array}{l}\text { Early (within } 1 \text { week) } \\
\quad \text { laparoscopic cholecystectomy }\end{array}$ & $\begin{array}{l}0.16(0.14-0.18) \\
\quad(p<0.0001)\end{array}$ & $\begin{array}{l}0.61(0.41-0.93) \\
\quad(p<0.0001)\end{array}$ & $\begin{array}{l}0.68(0.61-0.76) \\
\quad(p<0.0001)\end{array}$ & $0.47(0.42-0.53)(p<0.0001)$ \\
\hline Area under the ROC curve/c-index & 0.92 & 0.94 & 0.75 & 0.87 \\
\hline
\end{tabular}

Models are adjusted for patients' demographics (age, sex, race, income category, primary payer, and procedure days and type), hospital-level characteristics (hospital size, location, region, and teaching status), patients' comorbidity (hypertension, diabetes, obesity, hypercholesterolemia, drug, alcohol and tobacco abuse, alcohol withdrawal, HIV, renal dysfunction, ischemic heart disease, and chronic pancreatitis), complications (hypercalcemia, acute renal failure, shock, SIRS, ascites, pleural effusion, respiratory failure, and portal vein thrombosis), other procedures (ERCP, USG abdomen, CT abdomen, cholecystostomy, blood transfusion, parenteral nutrition), and Charlson's Comorbidity Index

$U L$, upper limit; $L L$, lower limit

*Morbidity: length of stay > 11 days (90th percentile) + discharge other than home

\# Discharge other than home: discharge to short-term hospital, skilled nursing facility, or intermediate care facility 
pancreatitis patients from the years 2003 to 2014 and associated factors such as outcomes (morbidity and mortality), cost of hospitalization, and other factors affecting it. In our knowledge, this is the first study that has studied and compared the effect of cholecystectomy done on a different time scale for index admission. Several national guidelines suggest that definitive treatment for gallstone pancreatitis is cholecystectomy within the same admission to the first few weeks to decrease the risk of recurrent biliary events $[3,12,13]$. However, nationwide audits from the USA and Europe have shown that most patients undergo cholecystectomy weeks or sometimes months after discharge from the hospital for mild biliary pancreatitis [14-16]. Sanjay et al. and Tyler et al. in their study discussed the difficulties in the implementation of treatment guidelines $[17,18]$. Data analysis of our study showed that $50.09 \%$ of total patients with gallstone pancreatitis had index admission laparoscopic cholecystectomy. Further analysis showed that the prevalence trend of immediate cholecystectomy increased from 32.6 to $49.8 \%$, and early and late cholecystectomy has decreased from (57.8 to $46.5 \%)$ and (9.5 to $3.5 \%$ ), respectively.

Younger patients (age 18-55) found to be having a higher prevalence of immediate laparoscopic cholecystectomy compared to older patients (age $>55$ years), which could be because of greater prevalence of comorbidities in older patients, in general, making them less suitable for surgery or more severe presentation with gallstone pancreatitis. Marc et al. observed that adherence to the current guidelines for the management of mild gallstone pancreatitis was lower in older adults with higher readmission rates. In his study, $33 \%$ of readmitted patients eventually required cholecystectomy. The author concluded that $40 \%$ of the patients who did not undergo the same admission (versus readmission interval) cholecystectomy would have benefited from definitive therapy in index admission [19]. The highest prevalence of immediate laparoscopic cholecystectomy is observed in Native Americans (48.8\%), and the least is seen in African-Americans (34.3\%). Interestingly, immediate cholecystectomy utilization prevalence is found to be higher in elective cholecystectomy admissions (7.24 versus 4.32 ) compared to non-elective cholecystectomy admission (92.76 versus 95.68). A weekend effect is also observed during analysis, in weekday's admission utilization prevalence of immediate cholecystectomy found to be higher (74.82 versus 70.73) compared to weekend admission ( 25.18 versus 29.27 ). The effect could be explained by a lack of available resource provisions such as magnetic resonance cholangiopancreatography (MRCP) and endoscopic retrograde cholangiopancreatography (ERCP), and inadequate specialist coverage and trained staff at weekends in many low resource institutes [20,21].

Patients with comorbidities, including ischemic heart disease, $\mathrm{CKD}$, diabetes, and hypertension, are observed to have a lower prevalence of immediate laparoscopic cholecystectomy compared to late laparoscopic cholecystectomy. Complication rates were lower in patients with an immediate index laparoscopic cholecystectomy group. Immediate cholecystectomy group patients had lower odds of mortality and morbidity and lower odds of requiring a short-term hospital, skilled nursing facility, or intermediate care facility at the time of discharge. The findings on low mortality are similar to the multicenter randomized controlled trial (PONCHO trial) by David da Costa et al., of 266 patients who underwent the same admission cholecystectomy within 3 days versus interval cholecystectomy in 25-30 days [22]. Lower prevalence of disability, length of stay, and cost of hospital utilization are seen in patients with immediate laparoscopic cholecystectomy as compared to patients with early and late laparoscopic cholecystectomy, which concur with multiple other clinical studies [23]. The cost of hospitalization is about three times higher in patients with late laparoscopic cholecystectomy compared to patients with immediate laparoscopic cholecystectomy. The cost-effective benefit of immediate cholecystectomy in acute gallstone pancreatitis has been illustrated in other studies as well $[24,25]$.

These findings do not establish any causative relationship between complications and the exact timing of surgery or related to the severity of pancreatitis because of the nature of the study. For a severe presentation of gallstone pancreatitis while almost always is a reason to delay the surgery, few studies have also observed some other limitations as well. A British study found only $70 \%$ compliance with the guideline's adherence because of inadequate imaging or inappropriate delay in diagnosis and noticed an increased risk of a recurrent biliary event. Delayed or non-availability of timely imaging test and intervention such as MRCP in low suspicion for common bile duct (CBD) stone or ERCP for high suspicion for $\mathrm{CBD}$ stone has also been factored in as a reason for delayed cholecystectomy [18, 26, 27]. Our study is one of the largest population-based studies to report the trends, outcomes, and complication profile of acute gallstone pancreatitis patients who underwent laparoscopic cholecystectomy in the same index hospitalization at different times. Though the NIS data is the largest national inpatient database with good statistical power, this study has limitations. This administrative database is obtained retrospectively by chart abstractions based on the discharge diagnosis codes and billing codes, and is susceptible to coding errors. Complications and outcomes depend on the severity of the disease. Our study as being based on the NIS data also has limitation of determining clinical disease severity difference between all the groups which may have affected the decision for timing of surgery. We have not followed up on the patient's post-hospitalization to evaluate the disability.

\section{Conclusion}

In our study, we found immediate laparoscopic cholecystectomy is better than early or delayed laparoscopic cholecystectomy, even in the same index admission. An inclusion of a 
definite timeline recommendation for immediate laparoscopic cholecystectomy (within $22 \mathrm{~h}$ ) in societal guidelines for management of acute biliary pancreatitis can provide further clarity and help improve adherence to guideline recommendations. Our study findings showed favorable outcome of immediate cholecystectomy, the study has many limitations as described in the "Discussion" section, and more prospective studies with large numbers of patients should be planned to evaluate the beneficial effects of immediate cholecystectomy in severe acute pancreatitis hospitalizations.

Supplementary Information The online version contains supplementary material available at https://doi.org/10.1007/s42399-021-00765-y.

Acknowledgements The author would like to acknowledge the help of Urvish Patel for Biostatistical analysis.

\section{Code Availability Yes}

Author Contribution AS conceptualized the idea. AS, AM, and JR reviewed the literature and collected the data with the help of SS and AJ. JR, AM, and SS formulated the tables. AS, SS, JR, and AJ wrote the main draft of the manuscript. AS supervised the project.

Data Availability Files for the raw analysis can be made available upon request.

\section{Compliance with ethical standards}

Ethics Approval We have read and have abided by the statement of ethical standards for manuscript submitted to the European Journal of Gastroenterology and Hepatology. The data has been taken from Nationwide Inpatient Sample, which is a deidentified database from the "Healthcare Utilization Project (HCUP)" sponsored by the Agency for Healthcare Research and Quality, so informed consent or IRB approval was not needed for the study. The relevant ethical oversight and HCUP Data Use Agreement were obtained for the study.

Conflict of Interest The authors declare no conflict of interest.

Open Access This article is licensed under a Creative Commons Attribution 4.0 International License, which permits use, sharing, adaptation, distribution and reproduction in any medium or format, as long as you give appropriate credit to the original author(s) and the source, provide a link to the Creative Commons licence, and indicate if changes were made. The images or other third party material in this article are included in the article's Creative Commons licence, unless indicated otherwise in a credit line to the material. If material is not included in the article's Creative Commons licence and your intended use is not permitted by statutory regulation or exceeds the permitted use, you will need to obtain permission directly from the copyright holder. To view a copy of this licence, visit http://creativecommons.org/licenses/by/4.0/.

\section{References}

1. Yadav D, Lowenfels AB. Trends in the epidemiology of the first attack of acute pancreatitis: a systematic review. Pancreas. 2006. https://doi.org/10.1097/01.mpa.0000236733.31617.52.
2. Forsmark CE, Baillie J. AGA Institute Technical Review on Acute Pancreatitis. Gastroenterology. 2007. https://doi.org/10.1053/j. gastro.2007.03.065.

3. Toouli J, Brooke-Smith M, Bassi C, Carr-Locke D, Telford J, Freeny $\mathrm{P}$, et al. Guidelines for the management of acute pancreatitis. J Gastroenterol Hepatol. 2002. https://doi.org/10.1046/j.14401746.17.s1.2.x.

4. Venneman NG, Renooij W, Rehfeld JF, VanBerge-Henegouwen GP, Go PMNYH, Breeders IAMJ, et al. Small gallstones, preserved gallbladder motility, and fast crystallization are associated with pancreatitis. Hepatology. 2005. https://doi.org/10.1002/hep.20616.

5. Hernandez V, Pascual I, Almela P, Añon R, Herreros B, Sanchiz V, et al. Recurrence of acute gallstone pancreatitis and relationship with cholecystectomy or endoscopic sphincterotomy. Am J Gastroenterol. 2004. https://doi.org/10.1111/j.1572-0241.2004. 40896.x.

6. Noel R, Arnelo U, Lundell L, Hammarqvist F, Jumaa H, Enochsson $\mathrm{L}$, et al. Index versus delayed cholecystectomy in mild gallstone pancreatitis: results of a randomized controlled trial. HPB. 2018. https://doi.org/10.1016/j.hpb.2018.03.016.

7. IAP/APA. Evidence-based guidelines for the management of acute pancreatitis. Pancreatology. 2013. https://doi.org/10.1016/j.pan. 2013.07.063.

8. Mueck KM, Wei S, Pedroza C, Bernardi K, Jackson ML, Liang MK, et al. Gallstone pancreatitis: admission versus normal cholecystectomy-a randomized trial (Gallstone PANC Trial). Ann Surg. 2019. https://doi.org/10.1097/SLA.0000000000003424.

9. Zhong FP, Wang K, Tan XQ, Nie J, Huang WF, Wang XF. The optimal timing of laparoscopic cholecystectomy in patients with mild gallstone pancreatitis: a meta-analysis. Medicine (United States). 2019. https://doi.org/10.1097/MD.0000000000017429.

10. Borreca D, Bona A, Bellomo MR, Borasi A, De Paolis P. Timing of cholecystectomy in acute biliary pancreatitis: is it still reasonable to wait? Minerva Chir. 2016.

11. Tenner S, Baillie J, Dewitt J, Vege SS. American College of Gastroenterology guideline: management of acute pancreatitis. Am J Gastroenterol. 2013. https://doi.org/10.1038/ajg.2013.218.

12. Johnson CD. UK guidelines for the management of acute pancreatitis. Gut. 2005. https://doi.org/10.1136/gut.2004.057026.

13. Banks PA, Freeman ML, Fass R, Baroni DS, Mutlu EA, Bernstein $\mathrm{DE}$, et al. Practice guidelines in acute pancreatitis. Am J Gastroenterol. 2006. https://doi.org/10.1111/j.1572-0241.2006. 00856.x.

14. Nguyen GC, Boudreau H, Jagannath SB. Hospital volume as a predictor for undergoing cholecystectomy after admission for acute biliary pancreatitis. Pancreas. 2010. https://doi.org/10.1097/MPA. 0b013e3181bd6387.

15. Monkhouse SJW, Court EL, Dash I, Coombs NJ. Two-week target for laparoscopic cholecystectomy following gallstone pancreatitis is achievable and cost neutral. Br J Surg. 2009. https://doi.org/10. 1002/bjs.6644.

16. Pezzilli R, Uomo G, Gabbrielli A, Zerbi A, Frulloni L, De Rai P, et al. A prospective multicentre survey on the treatment of acute pancreatitis in Italy. Dig Liver Dis. 2007. https://doi.org/10.1016/j. dld.2007.05.014.

17. Sanjay P, Yeeting S, Whigham C, Judson HK, Kulli C, Polignano FM, et al. Management guidelines for gallstone pancreatitis. Are the targets achievable? J Pancreas. 2009.

18. Stevens T, Parsi MA, Matthew WR. Acute pancreatitis: problems in adherence to guidelines. Cleve Clin J Med. 2009. https://doi.org/10. 3949/ccjm.76a.09060.

19. Trust MD, Sheffield KM, Boyd CA, Benarroch-Gampel J, Zhang D, Townsend CM, et al. Gallstone pancreatitis in older patients: are we operating enough? Surgery. 2011. https://doi.org/10.1016/j. surg.2011.07.072. 
20. Jehan F, Khan M, Kulvatunyou N, Hamidi M, Gries L, Zeeshan M, et al. Day of hospital admission and effect on outcomes: the weekend effect in acute gallstone pancreatitis. J Surg Res. 2019. https:// doi.org/10.1016/j.jss.2018.07.070.

21. Inamdar S, Sejpal DV, Ullah M, Trindade AJ. Weekend vs. weekday admissions for cholangitis requiring an ERCP: comparison of outcomes in a national cohort. Am J Gastroenterol. 2016. https:// doi.org/10.1038/ajg.2015.425.

22. Bouwense SA, Besselink MG, van Brunschot S, Bakker OJ, van Santvoort HC, Schepers NJ, et al. Pancreatitis of biliary origin, optimal timing of cholecystectomy (PONCHO trial): study protocol for a randomized controlled trial. Trials. 2012. https://doi.org/10. 1186/1745-6215-13-225.

23. Aboulian A, Chan T, Yaghoubian A, Kaji AH, Putnam B, Neville A, et al. Early cholecystectomy safely decreases hospital stay in patients with mild gallstone pancreatitis: a randomized prospective study. Ann Surg. 2010. https://doi.org/10.1097/SLA. 0b013e3181c38flf.

24. Morris S, Gurusamy KS, Patel N, Davidson BR. Cost-effectiveness of early laparoscopic cholecystectomy for mild acute gallstone pancreatitis. Br J Surg. 2014. https://doi.org/10.1002/bjs.9501.

25. da Costa DW, Dijksman LM, Bouwense SA, Schepers NJ, Besselink MG, van Santvoort HC, et al. Cost-effectiveness of same-admission versus interval cholecystectomy after mild gallstone pancreatitis in the PONCHO trial. Br J Surg. 2016. https:// doi.org/10.1002/bjs.10222.

26. Norton SA, Cheruvu CV, Collins J, Dix FP, Eyre-Brook IA. An assessment of clinical guidelines for the management of acute pancreatitis. Ann R Coll Surg Engl. 2001;83:399-405.

27. Lankisch PG, Weber-Dany B, Lerch MM. Clinical perspectives in pancreatology: compliance with acute pancreatitis guidelines in Germany [1]. Pancreatology. 2005. https://doi.org/10.1159/ 000087501.

28. McCormick PJ, Lin HM, Deiner SG, Levin MA. Validation of the All Patient Refined Diagnosis Related Group (APR-DRG) risk of mortality and severity of illness modifiers as a measure of perioperative risk. J Med Syst. 2018;42:81.

29. Baram D, Daroowalla F, Garcia R, Zhang G, Chen JJ, Healy E, et al. Use of the All Patient Refined-Diagnosis Related Group (APR-DRG) risk of mortality score as a severity adjustor in the medical ICU. Clin Med Circu Respir Pulm Med. 2008;2:19-25.

Publisher's Note Springer Nature remains neutral with regard to jurisdictional claims in published maps and institutional affiliations. 